\title{
Tire Mounting on a Car Using the Real-Time Control Architecture ARCADE
}

\author{
Thomas Nierhoff, Lei Lou, Vasiliki Koropouli, Martin Eggers, Timo Fritzsch, \\ Omiros Kourakos, Kolja Kühnlenz, Dongheui Lee, Bernd Radig, Martin Buss, \\ Sandra Hirche
}

\begin{abstract}
In comparison to industrial settings with structured environments, the operation of autonomous robots in unstructured and uncertain environments is more challenging. This video presents a generic control and system architecture ARCADE, applicable for real-time robot control in complex task situations. Several methods to cope with uncertainties are demonstrated with the example task of changing tires on a car. Approaches of object detection (applied to car, tires, and humans), robust real-time control of robot arms under perception uncertainty, and human-friendly haptic interaction are detailed. The video shows two robots jointly performing the task of mounting a mock-up tire to a real car using the proposed methods, realizing robust performance in an uncertain environment.
\end{abstract}

\section{INTRODUCTION}

Standard industrial robots have been widely used in automation and in particular in the automotive assembly domain for many years. Typically, such robots are highly specialized for a single task in a fixed position, and must be reprogrammed when changing the assembly tasks. In our project, we focus on a generic robot control and software architecture ARCADE, applicable in uncertain environments, that can autonomously assist humans to perform complex tasks [1]. ARCADE can seamlessly merge distributed perception, decision-making, and real-time control. For the example tire mounting scenario the following modules are included in the architecture: the approach in [2] generates safe trajectories in uncertain and dynamic environments; obstacle avoidance is performed using the method proposed in [3]; human-robot interaction and cooperation is realized using methods from [4]. ARCADE is extended for the task of mounting tires with the following modules: 1 . admittancebased position control combined with an estimator to suppress vibrations in closed kinematic chains; 2 . feature-based object recognition to cope with large pose variations; 3 . a distributed ceiling camera array to perceive the whole environment (intelligent space); 4. a module to transfer motions from humans and thus make the robot perform more human-like. In the presented video, a tire mounting task is displayed to demonstrate the flexibility and ease with which the ARCADE architecture can be adapted to different tasks.

\section{Control Architecture ARCADE}

ARCADE is an architecture for real-time control in multirobot systems. It has the following important features: hard real-time scheduling for feedback control; asynchronous

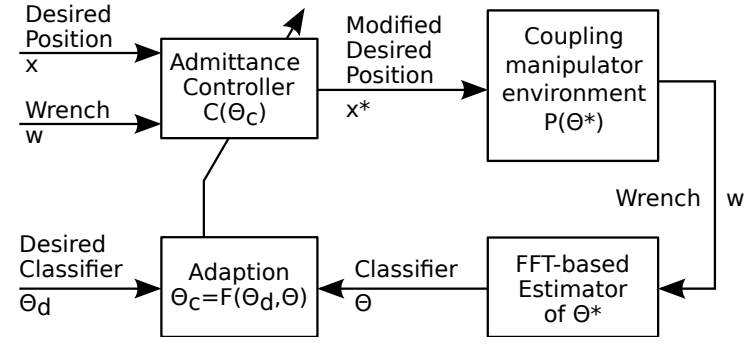

Fig. 1: Control scheme for vibration suppression.

remote procedure call for distributed modules; hardwarein-the-loop framework [5] for complex algorithm design. Different backends to ROS, Simulink/RTW are natively integrated. A toolkit for logging, debugging, and monitoring is implemented. The real-time robot arm controller is developed using Simulink/RTW, and runs under RTAI with a frequency of $1 \mathrm{KHz}$. It is connected with other modules in ARCADE.

The hardware components of the robots are two SICK LMS-200 laser range finders, one RGB-D camera, three i7-920 PCs, and two 7DoF anthropomorphic arms with two-finger grippers mounted on an omnidirectional platform (Fig. 3 left). On the ceiling 40 high-resolution cameras are mounted, and connected via Gigabit Ethernet to 40 computers to perceive the workshop of approximately $100 \mathrm{~m}^{2}$.

\section{Methods}

\section{A. Controller Design}

The arm control consists of an admittance-based position controller in combination with an inverse arm model [6]. As each end effector is fitted with a 6DoF JR3 force/torque sensor, the robot is able to detect the suspension based solely on haptic feedback when mounting the tire onto the car. To grasp and hold the tire, the admittance controller is used instead of a pure force control scheme. This provides greater robustness as internal/external forces do not have to be considered in the arm control loop. Still, oscillations do occur when holding the tire due to closed kinematic chains in combination with a finite sampling time and low damping/mass values of the arm admittance controller. An adaptive admittance controller is used in combination with an FFT-based estimator (Fig. 1), measuring oscillations of the end effector position and force data. When oscillatory behavior is detected the admittance damping values are increased and therewith the resonances no longer occur. 


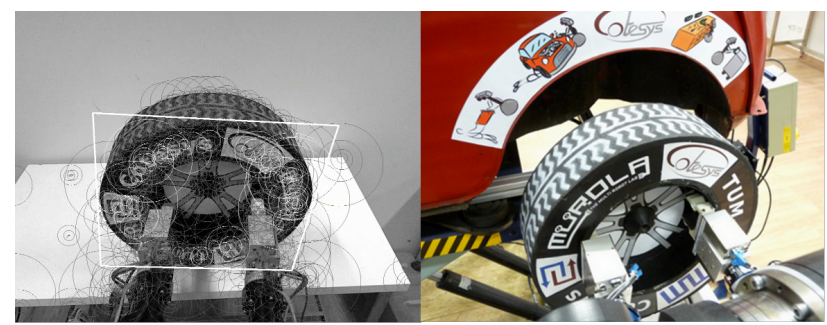

Fig. 2: Left: feature-based detection of the tire. Right: searching the axis of the car using admittance controller.

\section{B. Object Detection}

The main challenges in detecting the tires and the car are their large variations in scale and real-time pose estimation. The SURF [7] detector is employed to extract 2D multiscale features. To reduce the computational complexity, the FERNS [8] classifier is used instead of the SURF descriptor for feature matching (Fig. 2 left). After homography decomposition, the pose information is merged with depth information from a RGB-D sensor to improve the stability and accuracy of the image-based visual servoing. The sampling distribution in scale space is optimized [9] based on the octave-pyramid structure, thus the algorithm can detect the objects reliably in the 3 rd level octave with the base octave, corresponding to a 8 times scale variation.

\section{Environment Perception}

To estimate the positions of the humans cooperating with the robots, a planar binary code marker tracking-by-detection approach based on the OpenTL [10] framework is performed on images from the ceiling-mounted cameras (Fig. 3 right), which were calibrated using global optimization [11]. As targets are observed by several cameras simultaneously, data fusion is performed, where results obtained by cameras closer to the target receive higher weights to increase accuracy. The camera system reports $6 \mathrm{DoF}$ poses with an average error rate of about $1.5 \%$ (error/distance).

\section{Human-Robot Motion Transfer}

To make the robot movements look more human-friendly, we taught movements by imitation learning, using the methodology proposed in [12]. Motion data of 14 points on the human arms were captured by the Xsens Inertial Motion Capture suit. These motions were transferred onto the 7DoF robot arms. The motion transfer consists of a taskspace control approach where four task descriptors, two for the elbows and two for the hands, are tracked, and where the hands have a higher priority than the elbows.

\section{CONCLUSION}

This video shows two robots successfully mounting tires on a real car in an uncertain workshop environment using the generic control architecture ARCADE. Task performance is shown to be robust, and the robots can interact and collaborate with humans seamlessly. The admittance-based robot arm controller reduces vibrations in closed kinematic chains successfully. The feature-based detection can reliably

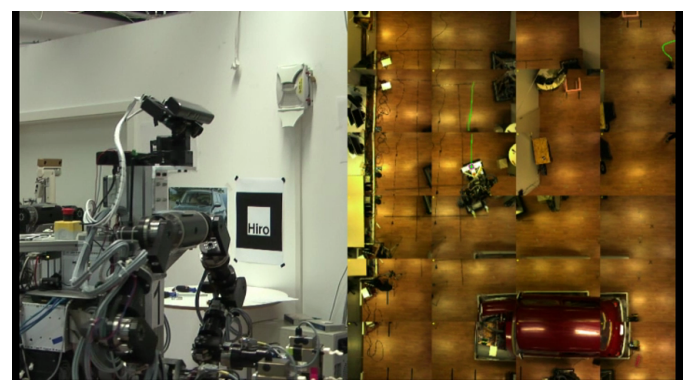

Fig. 3: Left: the robot platform. Right: the joint tiled view of 40 cameras observing the experimental area from a top-down perspective.

detect objects in the rather large workshop. A markerbased tracking module returns accurate human locations. The human motion is transferred to the robots to move their arms in a human-like way. In future work, the combination of the learned human motion primitives and the admittance controller will be investigated.

\section{ACKNOWLEDGMEnTs}

This work is supported in part by the DFG excellence initiative research cluster Cognition for Technical Systems CoTeSys, see also www. cotesys.org, the BMBF Bernstein Center for Computational Neuroscience Munich, see also www.bccn-munich.de, and the Institute for Advanced Study (IAS), Technische Universität München, see also www.tum-ias.de.

\section{REFERENCES}

[1] D. Althoff, O. Kourakos, M. Lawitzky, et al., "An architecture for real-time control in multi-robot systems," in Human Centered Robot Systems, pp. 43-52, Springer, 2009.

[2] D. Althoff, J. J. Kuffner, D. Wollherr, and M. Buss, "Safety assessment of robot trajectories for navigation in uncertain and dynamic environments," in ICRA, pp. $5407-5412,2011$.

[3] D. Althoff, C. N. Brand, D. Wollherr, and M. Buss, "Computing unions of inevitable collision states and increasing safety to unexpected obstacles," in IEEE/RSJ International Conference on Intelligent Robots and Systems, pp. $3114-3119,2011$.

[4] M. Lawitzky, J. M. Hernandez, and S. Hirche, "Rapid prototyping of planning, learning and control in physical human-robot interaction," 13th International Symposium on Experimental Robotics (ISER), pp. $819-824,2012$.

[5] L. Lou, Hardware-in-the-Loop Extension of RTAI for Simulink/RTW. tech. rep., Institute of Automatic Control Engineering, TUM, 2008.

[6] E. Lutscher, M. Lawitzky, and S. H. G. Cheng, "A control strategy for operating unknown constrained mechanisms," in ICRA, pp. 819 824, 2011.

[7] H. Bay, T. A. Ess, Tuytelaars, and L. V. Gool, "Surf: Speeded up robust features," in Computer Vision and Image Understanding (CVIU), vol. 110 , pp. 346-359, 2008.

[8] M. Calonder, V. Lepetit, and P. F. M. Ozuysal, "Fast keypoint recognition using random ferns," in IEEE Transactions on Pattern Analysis and Machine Intelligence, vol. 32, pp. 448-461, 2010.

[9] L. Lou, "High sampling rate visual servoing based on distributed computation over the network," Master's thesis, TUM, 2010.

[10] G. Panin, Model-based visual tracking: the OpenTL framework. Wiley, 2011.

[11] M. Eggers, V. Dikov, C. Steger, and B. Radig, "Setup and calibration of a distributed camera system for surveillance of laboratory space," in 8th Open German-Russian Workshop on Pattern Recognition and Image Understanding, pp. 44-47, 2011.

[12] B. Dariush, M. Gienger, B. Jian, C. Goerick, and K. Fujimura, "Whole body humanoid control from human motion descriptors," in International Conference on Robotics and Automation, pp. 2677 2684, 2008. 